\title{
Immunologic timeline of Ebola virus disease and recovery in humans
}

\author{
Anita K. McElroy, ${ }^{1,2}$ Rama S. Akondy, ${ }^{3}$ David R. Mcllwain, ${ }^{4}$ Han Chen, ${ }^{4}$ Zach Bjornson-Hooper, ${ }^{4}$ \\ Nilanjan Mukherjee, ${ }^{4}$ Aneesh K. Mehta, ${ }^{3}$ Garry Nolan, ${ }^{4}$ Stuart T. Nichol, ${ }^{1}$ \\ and Christina F. Spiropoulou ${ }^{1}$ \\ 'Viral Special Pathogens Branch, Centers for Disease Control and Prevention, Atlanta, Georgia, USA. ²Division of \\ Pediatric Infectious Diseases and Center for Vaccine Research, University of Pittsburgh School of Medicine, Pittsburgh, \\ Pennsylvania, USA. ${ }^{3}$ Division of Infectious Diseases, Emory University School of Medicine, Atlanta, Georgia, USA. “Baxter \\ Laboratory for Stem Cell Biology, Department of Microbiology and Immunology, Stanford University School of Medicine, \\ Stanford, California, USA.
}

\begin{abstract}
A complete understanding of human immune responses to Ebola virus infection is limited by the availability of specimens and the requirement for biosafety level 4 (BSL-4) containment. In an effort to bridge this gap, we evaluated cryopreserved PBMCs from 4 patients who survived Ebola virus disease (EVD) using an established mass cytometry antibody panel to characterize various cell populations during both the acute and convalescent phases. Acute loss of nonclassical monocytes and myeloid DCs, especially CD1c ${ }^{+}$DCs, was noted. Classical monocyte proliferation and CD38 upregulation on plasmacytoid DCs coincided with declining viral load. Unsupervised analysis of cell abundance demonstrated acute declines in monocytic, NK, and T cell populations, but some populations, many of myeloid origin, increased in abundance during the acute phase, suggesting emergency hematopoiesis. Despite cell losses during the acute phase, upregulation of Ki-67 correlated with recovery of cell populations over time. These data provide insights into the human immune response during EVD.
\end{abstract}

Conflict of interest: The authors have declared that no conflict of interest exists.

Copyright: (c) 2020, American Society for Clinical Investigation.

Submitted: February 17, 2020

Accepted: April 22, 2020

Published: May 21, 2020.

Reference information: /CI Insight. 2020;5(10):e137260.

https://doi.org/10.1172/jici.

insight.137260.

\section{Introduction}

Ebola virus (EBOV) infection in humans is characterized by severe, often fatal disease. Various factors have correlated with a fatal outcome, including patient age, high viral load, a dysregulated inflammatory response, endothelial dysfunction, coagulopathy, lymphocyte apoptosis, lack of $\mathrm{T}$ cell receptor diversity, declines in NK and monocyte populations, presence of inhibitory markers on activated $\mathrm{T}$ cells, and inadequate adaptive immune responses (1-13). Notably, the pathophysiology of Ebola virus disease (EVD) seems to center around the host immune response. Studies of the human immune response during EVD have expanded significantly since the 2014 outbreak in West Africa. However, conducting studies using primary human samples obtained from patients with EVD remains challenging, given that outbreaks typically occur in resource-limited settings, and handling such samples requires appropriate biological containment.

One way in which researchers have circumvented the difficulties surrounding the use of primary human samples from patients with EVD is by exposing human immune cells to EBOV in vitro to understand how viral infection alters cell function. The report of direct infection of DCs and macrophages in EBOV-infected cynomolgus macaques led many research groups to focus on cells of myeloid origin (14). EBOV productively infects monocytes and monocyte-derived macrophages in vitro, stimulates production of inflammatory cytokines, and actively inhibits IFN- $\alpha$ production by these cells $(15,16)$. At least some of these effects may be mediated via binding of EBOV glycoprotein (GP) to TLR4 on the surface of macrophages (17). However, monocyte-derived DCs, while able to support viral replication, do not produce inflammatory cytokines and are impaired in their ability to stimulate T cells $(18,19)$. Importantly, when myeloid DCs (mDCs) and plasmacytoid DCs (pDCs) were purified directly from human blood rather than generated in vitro, the mDCs were competent for viral replication, while the pDCs were not (20). Furthermore, pDCs were shown to be resistant to the anti-interferon activity of the EBOV VP35 protein (21). pDCs are the major producers of IFN- $\alpha$ and the drivers of downstream adaptive immunity (22). Since it is well established that $\mathrm{T}$ cell activation occurs during human $\operatorname{EVD}(4,23)$, the status of pDCs in patients with EVD is an unexplored area of importance. 
Another important type of immune cell that has been the focus of intense study following EBOV exposure is the T cell. Lymphocyte apoptosis has been noted in patients with EVD (10) and reported in vivo in both mouse $(24,25)$ and nonhuman primate (26) models of EVD. In vitro studies have additionally demonstrated lymphocyte apoptosis, specifically of T cells, upon exposure to EBOV (27), despite the lack of productive T cell infection by EBOV. This effect is mediated at least in part by the detection of EBOV GP by T cell TLR4 (28).

The effects of EBOV on NK cells and B cells are less well understood. Decline in NK cell numbers and certain killer cell immunoglobulin-like receptor (KIR) genotypes is associated with a fatal outcome in patients with $\operatorname{EVD}(1,29)$, but the mechanism of NK cell loss and function of these cells during infection is unknown. Individuals infected with EBOV have a marked plasmablast and activated B cell response and have been shown to produce high-affinity antibodies directed against the viral GP during convalescence $(23,30,31)$.

To date, each study of cellular immunity in patients with EVD has focused on a specific cell population. This is in part due to the limitations of flow cytometry, which can typically only handle up to 14 different parameters simultaneously depending on the instrument. This limitation can be overcome by using mass cytometry, which permits substantially more multiplexing. Mass cytometry is a method that uses antibodies labeled with heavy metal tags instead of fluorophores to identify various cell populations according to their marker expression. Labeled cells are detected using time-of-flight mass spectrometry, so the technology is often referred to as CyTOF (32). This method has been used to investigate the human immune system in both natural and disease states (33-38). These high-dimensional analyses offer insight into an immune response that may be missed by detection markers spanning only limited cell populations. In this study, we use mass cytometry to gain a broad yet in-depth overview of the human immune response to EVD.

In the fall 2014, 4 patients with EVD received care in the Emory University Hospital Serious Communicable Diseases Unit (39-42). The close proximity of the CDC, which houses a biosafety level 4 (BSL-4) laboratory with approval for conducting work on EBOV, permitted the cryopreservation of PBMCs from these patients. The availability of these samples combined with a validated mass cytometry antibody panel for human use and the fortuitous presence of a CyTOF mass cytometer on the Emory University campus provided us with the opportunity to identify the consequences of EBOV infection on the human immune system in patients with EVD. In this article, we describe the major findings and, importantly, make the data set available for other investigators to explore.

\section{Results}

Sample selection and panel validation. Two time points were selected for each of the patients identified as EVD2, EVD5, and EVD15. The first was the earliest sample available for each patient; in all cases, the patients were still viremic at this point. The second time point chosen was when the patient was clinically in convalescence and when viral load was either markedly lower (in the case of EVD2) or undetectable (EVD5 and EVD15). In these 3 patients, the viral load was clearly declining between the first and second time points, so the first time point was considered to be one in which viral load control had been achieved. EVD9 had more samples collected over a longer period of time. Importantly, for EVD9, samples were available before viral peak, so samples were chosen before and after viral load control was achieved and well into convalescence. Table 1 presents the viral load in each patient sample over time. Samples used in this study are shown in bold. Patient identification and sample dates are identical to those in previously published studies for comparison $(8,23,30,31)$.

Given the scarcity of PBMCs from patients with EVD, we wanted to use an established mass cytometry panel for these studies rather than developing and validating a new panel. The panel chosen was developed for use in human immunology studies $(43,44)$. It contains of a series of surface antibodies for phenotypically classifying cells and a series of phospho-specific antibodies for evaluating intracellular signaling pathways (see Supplemental Table 1 for antibody details; supplemental material available online with this article; https:// doi.org/10.1172/jci.insight.137260DS1). Lyophilized aliquots of the validated panel for both surface and intracellular antibodies were first tested on control PBMCs to ensure that the panel performance was consistent using the CyTOF machine at Emory University and that the required fixation for removing EBOV samples from BSL-4 did not alter the results. Signal quality was maintained under these conditions for all markers with the notable exception of CCR7, so this marker was excluded from analysis (data not shown).

Gating strategy and analysis of various cell populations. Each patient sample tube contained cryopreserved PBMCs that were derived from $2 \mathrm{~mL}$ whole blood. For control PBMC samples, $2 \times 10^{6}$ cells were used per tube. The process of preparing PBMCs removes RBCs, neutrophils, and platelets from 
Table 1. Patient samples as a function of time and viral load

\begin{tabular}{|c|c|c|c|c|}
\hline \multirow{2}{*}{ Day after symptom onset } & \multicolumn{4}{|c|}{ Donor } \\
\hline & EVD2 & EVD5 & EVD9 & EVD15 \\
\hline 2 & & & & $1.89 \times 10^{2}$ \\
\hline 3 & & & & $6.78 \times 10^{1}$ \\
\hline 4 & & & & ND \\
\hline 5 & & & $6.09 \times 10^{6}$ & \\
\hline 6 & & & $1.41 \times 10^{7}$ & ND \\
\hline 9 & & & $3.17 \times 10^{7}$ & ND \\
\hline 10 & & & $1.65 \times 10^{7}$ & ND \\
\hline 11 & & & $6.04 \times 10^{6}$ & ND \\
\hline 12 & $5.54 \times 10^{4}$ & & $3.89 \times 10^{6}$ & \\
\hline 13 & $4.08 \times 10^{3}$ & & $3.42 \times 10^{5}$ & \\
\hline 18 & & ND & $9.98 \times 10^{1}$ & \\
\hline 19 & $6.30 \times 10^{1}$ & & $2.42 \times 10^{2}$ & \\
\hline 20 & $3.11 \times 10^{1}$ & ND & $3.33 \times 10^{1}$ & \\
\hline 21 & & ND & ND & \\
\hline 22 & ND & & $1.50 \times 10^{2}$ & \\
\hline 23 & & ND & & \\
\hline 24 & ND & & ND & \\
\hline 25 & & ND & ND & \\
\hline 26 & ND & & ND & \\
\hline 27 & & ND & ND & \\
\hline 28 & ND & & ND & \\
\hline
\end{tabular}

Cells with no content indicate that no sample was available on that date. Values represent viral load. ND, not detected. Boldface indicates samples that were used in this study.

the sample, so very few of these events are noted, but they were still gated out for downstream analysis. A standard gating strategy (Figure 1) was used to define B cells $\left(\mathrm{CD} 19^{+}\right), \mathrm{T}$ cells $\left(\mathrm{CD}^{+}\right), \mathrm{NK}$ cells $\left(\mathrm{CD} 3^{-} \mathrm{CD} 19^{-} \mathrm{CD} 7^{+} \mathrm{CD} 56^{+}\right)$, NKT cells $\left(\mathrm{CD}^{+} \mathrm{CD} 161^{+} \mathrm{CD} 56^{+} \mathrm{CD} 16^{+}\right)$, basophils $\left(\mathrm{CD}^{-} \mathrm{CD} 19^{-}\right.$ CD123 ${ }^{+}$HLA-DR-), monocytes ( Lin-CD14 $^{+}$and/or CD16 ${ }^{+}$), and DCs (Lin-CD14-CD16-HLA-DR ${ }^{+}$). B cells were further classified as plasmablasts $\left(\mathrm{CD} 38^{+} \mathrm{CD} 27^{+}\right)$, naive $\left(\mathrm{CD} 27^{-}\right)$, or memory $\left(\mathrm{CD} 27^{+}\right)$, and subgrouped as class-switched $\left(\mathrm{IgM}^{-}\right)$or non-class switched $\left(\mathrm{IgM}^{+}\right)$. T cells were classified as $\mathrm{CD} 4^{+}$or $\mathrm{CD} 8^{+}$, and activated cells were defined as $\mathrm{Ki}-67^{+} \mathrm{CD} 38^{+}$. NK cells were classified as $\mathrm{CD} 16^{+}$or $\mathrm{CD} 16^{-}$. Monocytes were classified as classical $\left(\mathrm{CD} 14^{+} \mathrm{CD} 16^{-}\right)$, intermediate $\left(\mathrm{CD} 14^{+} \mathrm{CD} 16^{+}\right)$, or nonclassical $\left(\mathrm{CD} 14^{-} \mathrm{CD} 16^{+}\right)$. DCs were further defined as being plasmacytoid $\left(\mathrm{pDC}, \mathrm{CD} 123^{+}\right)$or myeloid $(\mathrm{mDC}$, $\left.\mathrm{CD} 11 \mathrm{c}^{+}\right)$. From this gating strategy, the frequency of each cell type in each sample was determined as a function of non-neutrophil $\mathrm{CD} 45^{+}$events (Figure 2A). Patient samples are shown in comparison to the range obtained from the control samples (gray shading). $\mathrm{CD} 4^{+} \mathrm{T}$ cells were activated in all patients, as previously published. One striking feature of $\mathrm{CD} 8^{+} \mathrm{T}$ cell activation was the decreased frequency of activated $\mathrm{CD} 8^{+} \mathrm{T}$ cells in EVD9 relative to the other patients. This has relevance as a predictor of clinical outcome, as other groups have shown an association between $\mathrm{T}$ cell function and survival (3). Indeed, patient EVD9 was seriously ill and would not have survived without intensive care support, while the other 3 patients in the cohort had more moderate disease $(40,42)$. Importantly the decrease in activated $\mathrm{CD} 8^{+} \mathrm{T}$ cells in EVD9 was only appreciated because here, activated $\mathrm{CD} 8^{+} \mathrm{T}$ cells were enumerated as a function of non-neutrophil $\mathrm{CD} 45^{+}$events rather than as a proportion of total $\mathrm{T}$ cells, as done previously. In the prior analysis, the magnitude of $\mathrm{CD} 8^{+} \mathrm{T}$ cell activation as a function of total $\mathrm{T}$ cells was similar 

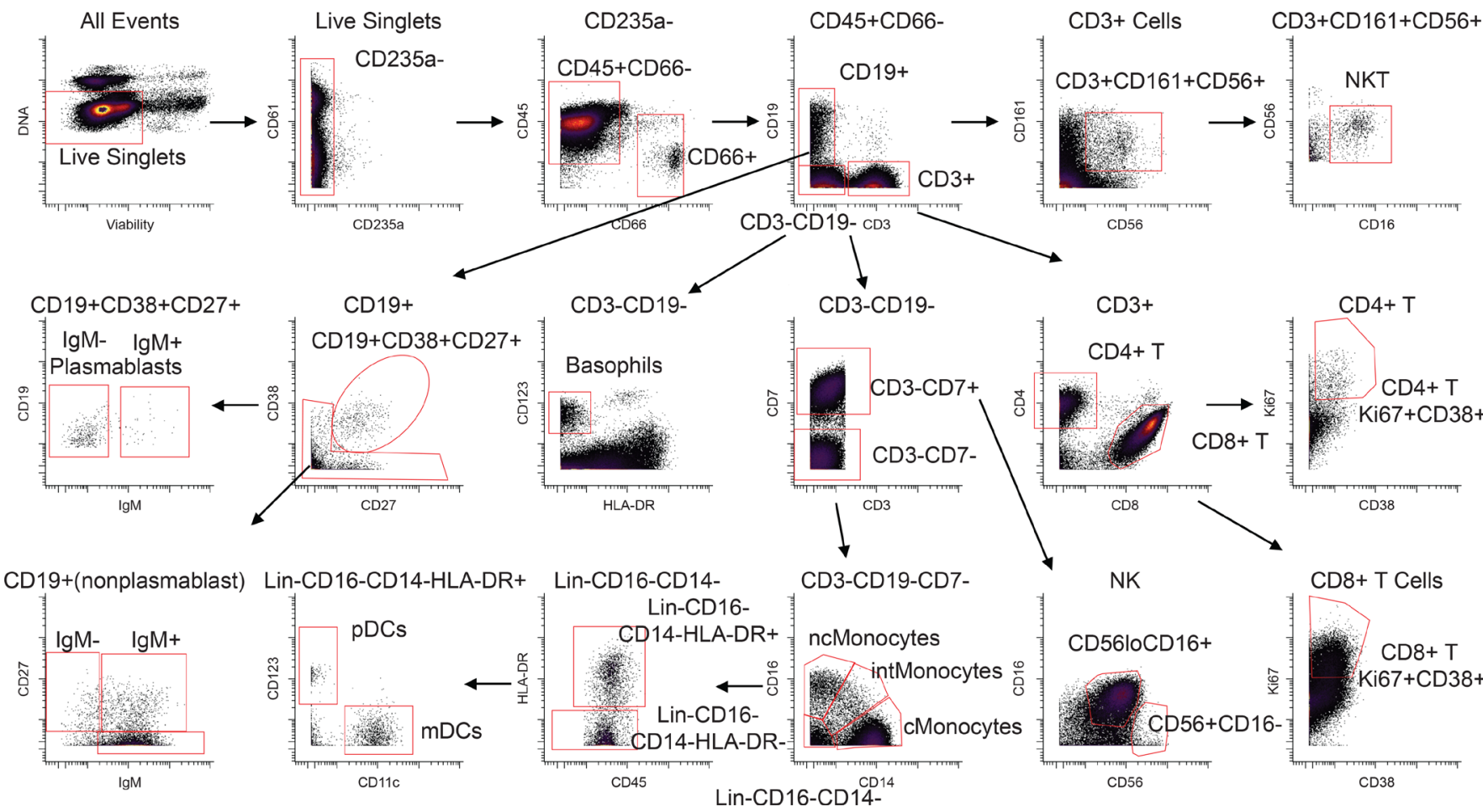

Figure 1. Gating strategy. The gating strategy is represented using the day 25 sample from patient EVD5. Common PBMC populations are identified. cMonocytes, classical monocytes; intMonocytes, intermediate monocytes; ncMonocytes; nonclassical monocytes.

across all donors (23), and we found a comparable activation of $\mathrm{CD} 8^{+} \mathrm{T}$ cells as a function of total $\mathrm{T}$ cells by CyTOF in present study (data not shown). Using an enumeration based on $\mathrm{CD} 45^{+}$events also revealed a previously unappreciated cell population. This occurs because the cell population that makes up the extra $\mathrm{CD} 45^{+}$events in EVD9 remains largely unaccounted for in traditional gating since it lacks lineage markers, but in our analysis was found to be $\mathrm{CD}_{11 b^{+}}, \mathrm{Ki}_{-}-67^{+}$, and $\mathrm{pSTAT3}^{+}$(Figure 2B). In EVD9, this population ranged from $1 \%$ to as much as $17 \%$ (on day 14 after symptom onset) of all of the gated $\mathrm{CD} 45^{+}$events. The other $3 \mathrm{EVD}$ patients also had this cell population, albeit at much lower levels than EVD9 (data not shown). Importantly, control patients did not have substantial quantities of this cell population, and EVD9 had the most. This suggests that the population could have relevance to disease, since EVD9 was severely ill, required extracorporeal support, and would not have survived without intensive critical care. All 4 patients also received experimental therapeutics, some of which could have altered their immune responses (23).

The frequency of basophils was elevated in several of the patients at different time points as compared with controls. NKT cells were largely in the control range, and NK cells were low in 1 patient but normal or elevated in the others. Increased proliferation, as evidenced by Ki-67 staining in both NKT and NK cells, was noted in acute-phase samples from all patients (Figure 2A).

As previously reported, plasmablasts were increased in all 4 patients, most prominently in EVD5 at 17 days after symptom onset. Notably, EVD5 was also shown to have the highest numbers of EBOV GP-specific memory B cells in a detailed analysis of the B cell responses of these 4 patients (30). In all patients, samples from all time points contained more class-switched plasmablasts than $\operatorname{IgM}^{+}$plasmablasts. Frequencies of naive, class-switched memory, and non-class-switched memory B cells were similar to those of controls.

Overall frequencies of classical monocytes were elevated in 3 of the 4 patients at the times sampled, while intermediate and nonclassical monocytes were in the normal range when considered as a frequency of $\mathrm{CD} 45^{+}$events. Using frequencies of non-neutrophil $\mathrm{CD} 45^{+}$events underestimates the dramatic shifts that occur in these populations during the course of the infection (Figure 3A, graphs). Previous work by others had demonstrated decreased numbers of $\mathrm{CD} 16^{+}$monocytes in association with fatal EVD (2), so we evaluated monocytes over time in this cohort of patients. In all patients, an almost complete loss of 
A $\mathrm{CD} 3+\mathrm{CD} 4+\mathrm{Ki}-67+\mathrm{CD} 38+$
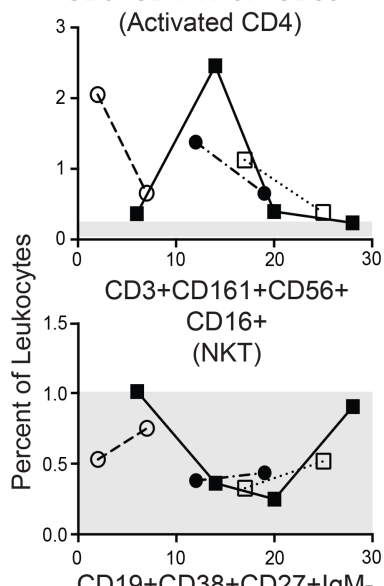

CD19+CD38+CD27+lgM(Class switched plasmablasts)

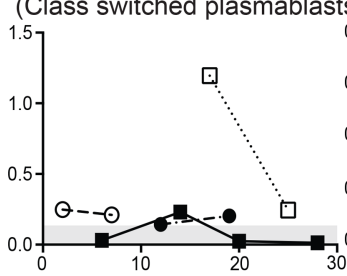

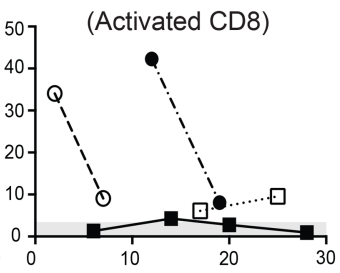
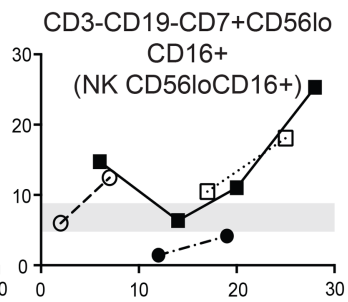

CD19+CD38+CD27+lgM+ (lgM+ plasmablasts)
CD3-CD19-CD123+HLADR-

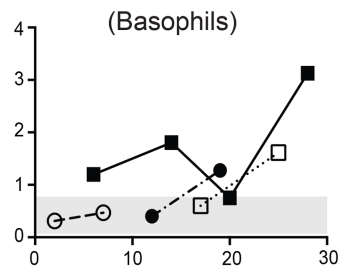

CD3-CD19-CD7+CD56+

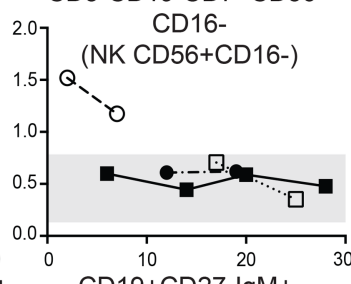

CD19+CD27-lgM+

(Naive B Cells)

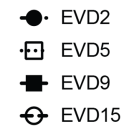

EVD2

- EVD9

๑) EVD15

Ki-67

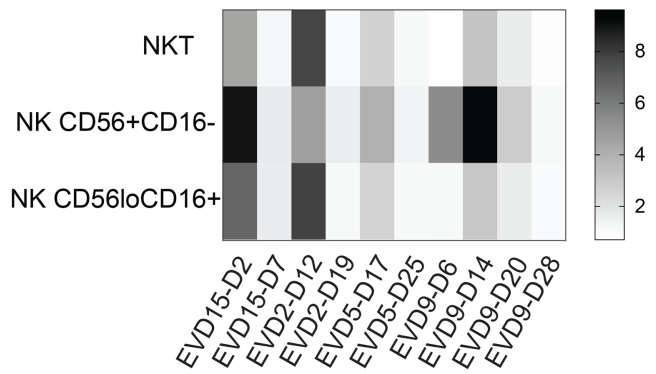

CD19+CD27+lgM-

CD19+CD27+lgM+ (Class switched memory B) (Non-class switched memory B)

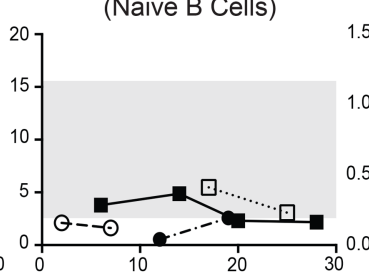

Days after symptom onset
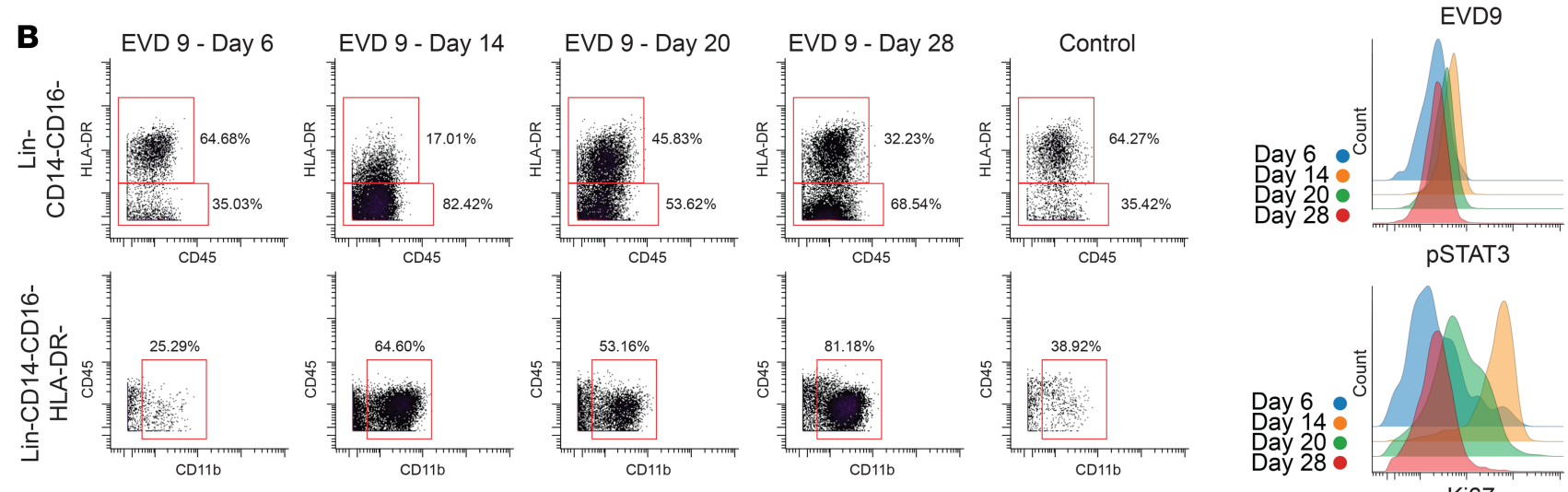

Ki67

Figure 2. Frequencies and activation status of various cell populations. (A) Frequencies of T cells, B cells, basophils, and NK cells as a percentage of CD45+CD66- leukocytes in patients compared with normal healthy controls (range for all controls in gray). Fold increase in median Ki-67 signal as compared with that in a control population is shown for NK and NKT cells at each patient time point. D2, day 2. (B) The expanded Lin-CD14-CD16-HLA-DR- but CD116 ${ }^{+}$ population that was noted in EVD9 is shown, with histograms demonstrating pSTAT3 and Ki-67 expression in these cells.

nonclassical and intermediate monocytes was noted at the earliest time point evaluated (Figure 3A, flow plots), followed by repopulation of first intermediate and then nonclassical monocytes. Notably, this was accompanied by a marked increase in proliferation of classical monocytes that preceded the appearance of intermediate monocytes (Figure 3B).

$\mathrm{mDC}$ levels were lower than normal at the first time point evaluated in all 4 patients (Figure $4 \mathrm{~A}$, graphs). The dramatic loss of $\mathrm{mDCs}$ that occurred during the acute phase was more readily appreciated by visualizing raw flow plots (Figure $4 \mathrm{~A}$ ) and was most prominent in the first sample from EVD9, which represents a time in the infection when viral load control had not yet been achieved. Following viral load control and into convalescence, the $\mathrm{mDC}$ population returned to control levels in all patients. Subsets of $\mathrm{mDC}$ sere also evaluated, and $\mathrm{CD} 1 \mathrm{c}^{+} \mathrm{mDCs}$ showed the most striking decline during the acute phase, with robust return and increased expression of $\mathrm{CD} 1 \mathrm{c}$ on $\mathrm{mDCs}$ during convalescence (Figure 4B). CD141 ${ }^{+}$ $\mathrm{mDC}$ s were also evaluated, but an insufficient number were identified in all patients at all time points to make any conclusions regarding this subset. 
pDC frequencies were elevated above control levels only in EVD9 (Figure 4C), and only at the first time point. Perhaps the marked increase in pDCs in this patient was related to the severity of disease, as at that time point, the patient had all of the clinical, virologic, and laboratory indicators of a fatal outcome. Alternatively, elevated numbers of pDCs could have been secondary to innate immune stimulation of the experimental therapeutic (siRNA) that they received. While not all patients exhibited an absolute increase in $\mathrm{pDC}$ numbers, in 3 of the 4 patients, CD38 was upregulated on pDCs at the earliest time point. Importantly, pDCs were observed at all time points and were activated, as evidenced by the expression of the activation marker CD38.

SCAFFoLD maps of cell abundance identify unique cell populations that are expanded during the acute phase of infection. Multiparameter analyses can also be visualized with an unsupervised approach that can identify similarities and differences in cell populations that are not revealed by traditional gating. Single-cell analysis by fixed force- and landmark-directed (SCAFFoLD) (45) maps were created to illustrate the abundance of various cell populations at the acute versus convalescent time point for each patient (Figure 5A). For EVD9, the time points of day 14 and day 28 were chosen because these were most similar to the time points available from the other patients in previous analyses (phenotypic properties and viral control). Most cells were of lower abundance during the acute phase of disease. However, select cell populations were more abundant during the acute phase, most notably near the monocyte or CD8 ${ }^{+} \mathrm{T}$ cell cluster. For example, cluster 41, seen in increased abundance in all 4 patients, is a $\mathrm{Lin}^{-} \mathrm{CD} 11 \mathrm{~b}^{+} \mathrm{Ki}-67^{+} \mathrm{pSTAT} 1^{+}$population. Cluster 98, in high abundance in the acute phase in 3 of the 4 patients, is $\mathrm{CD} 4^{+} \mathrm{CD} 8^{+} \mathrm{Ki}-67^{+} \mathrm{pSTAT} 1^{+}$. Double-positive $\mathrm{T}$ cells have been reported in other infectious disease processes (46), although their significance is unclear. SCAFFoLD maps of the Ki-67 signal were also generated for all 4 patients (Figure 5B), demonstrating that despite the low abundance of many cell populations at the acute phase, broad, active proliferation also occurred, which may have contributed to normalization of cell numbers over time.

Intracellular signaling pathways. An added benefit of the mass cytometry antibody panel (43) repurposed for this study of patients with EVD is the spectrum of signaling pathways that are assessed using phospho-specific antibodies. The various cell populations that were defined by traditional gating (Figure 1) were evaluated for median signal intensity of each intracellular marker. Values were compared with the median signal intensity for the simultaneously acquired control samples. Fold change in median signal of samples versus controls is represented in heatmaps (Supplementary Figure 1). Increased activity in multiple pathways was noted in various B cell populations from patients with EVD. Caution must be used in interpreting these data, since the samples were not prepared specifically for this analysis, and no phosphatase or kinase inhibitors were included when the samples were obtained or processed for cryopreservation. However, all samples (EVD and controls) were handled similarly, so the marked increases in pSTAT1 observed in naive B cells in 3 of the 4 patients and of pSTAT5 in class-switched plasmablasts seen at later time points in all 4 patients are likely worth noting.

\section{Discussion}

In this study, we report changes that occurred in human PBMCs during EBOV infection. Use of mass cytometry for the analyses permitted the application of a large multiparameter antibody panel. This data set provides insights into the human immune response to EBOV infection in vivo. Importantly, published observations from previous work by our group and others using traditional flow cytometry are consistent with our results.

Monocyte loss and repopulation. The dramatic loss of intermediate and nonclassical monocytes was striking in all 4 patients during the acute phase, in line with prior observations of monocyte loss in patients with EVD (2). EBOV infects monocytes in vitro, so it is possible that these cells were lost to infection-related cell death. However, $\mathrm{CD} 16^{+}$monocytes are known to play a unique role in patrolling and responding to inflammation, so they may be lost from blood due to recruitment to the inflamed tissues (47). While the full function of intermediate monocytes has not been clearly defined, they are believed to play a role in $\mathrm{T}$ cell proliferation and stimulation, inflammatory responses, and presentation of antigen (47). Recently, a similar phenomenon was reported to occur during dengue virus infection: intermediate monocytes were lost and then repopulated via upregulation of CD16 on classical monocytes (48). Our finding that intermediate monocytes repopulate first, followed by nonclassical monocytes after marked classical monocyte proliferation, would be consistent with that phenomenon occurring during EVD. Notably, a study performed in humans infected with Chikungunya virus (49) made use of mass cytometry to define and characterize the changes that occur during acute infection and recovery; an expansion of the intermediate monocyte 
A

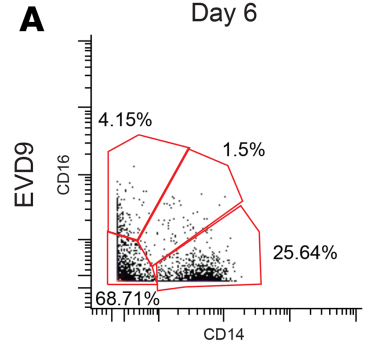

Day 12

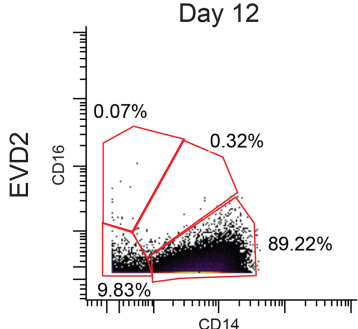

Day 17

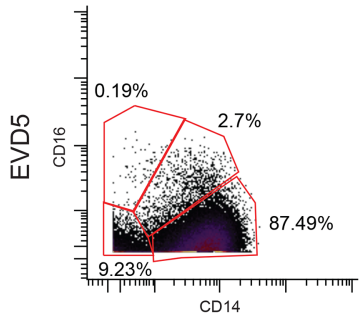

Day 2

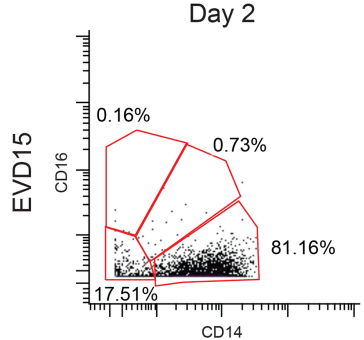

B

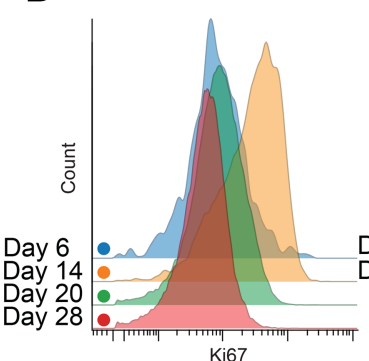

Day 14

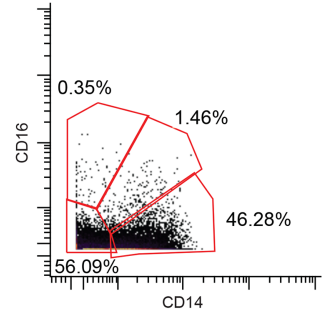

Day 19

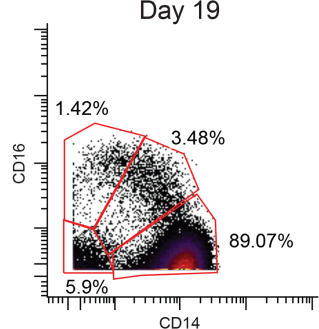

Day 25

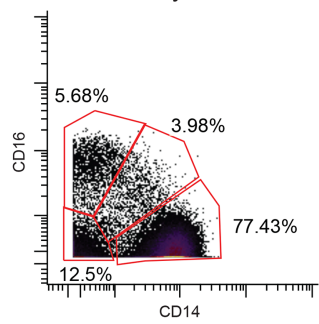

Day 7

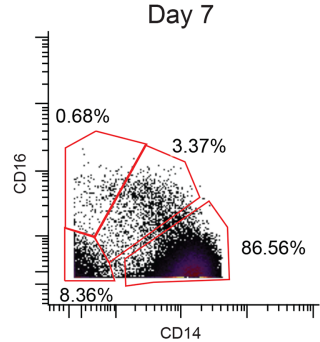

EVD2

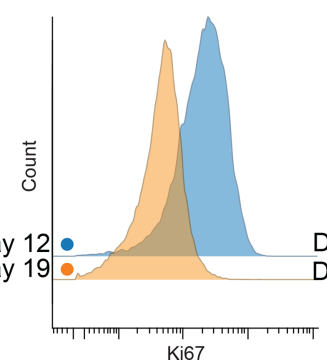

Day 20

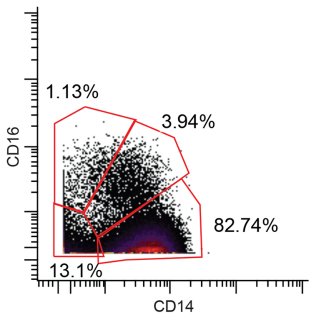

Day 28

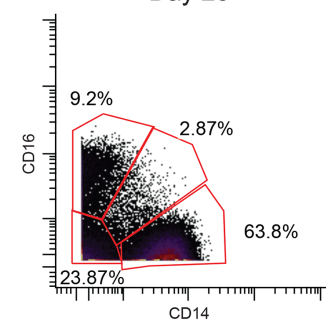

Lin-CD14+CD16-

(cMonocytes)

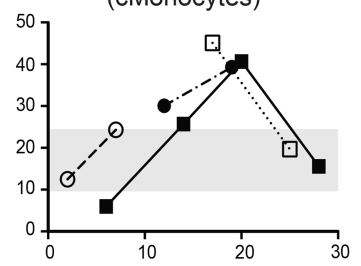

Lin-CD14+CD16+

(intMonocytes)

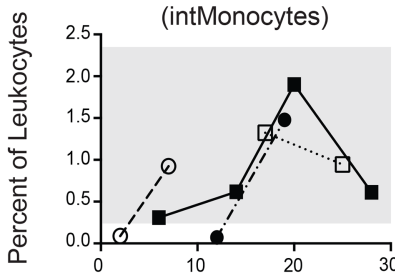

Lin-CD14-CD16-

(ncMonocytes)

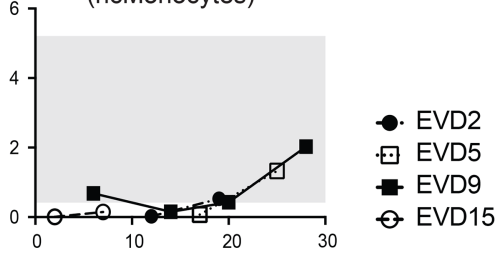

Days Post Symptom Onset

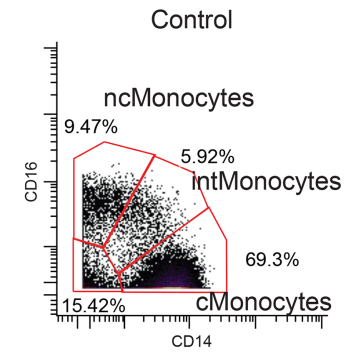


A

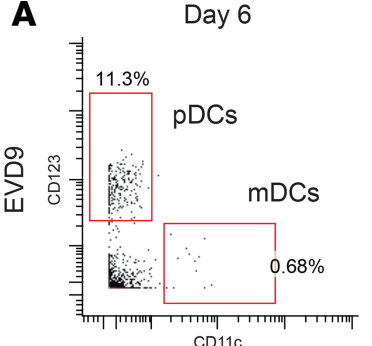

Day 12

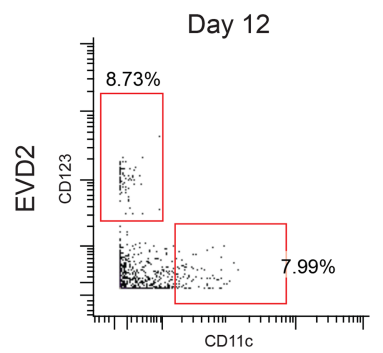

Day 17

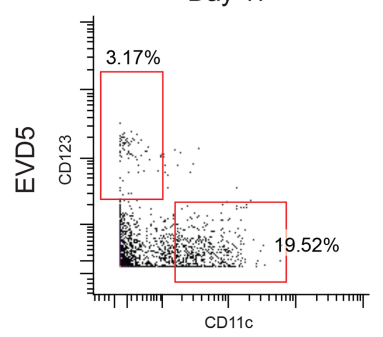

Day 2

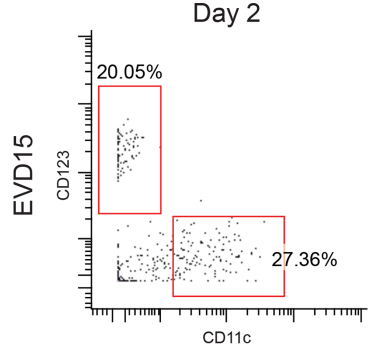

Lin-CD16-CD14-

HLA-DR+CD11c+

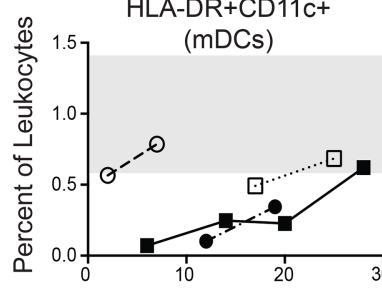

Day 14

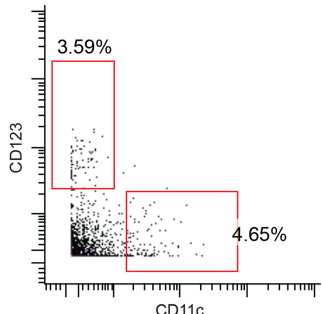

Day 19

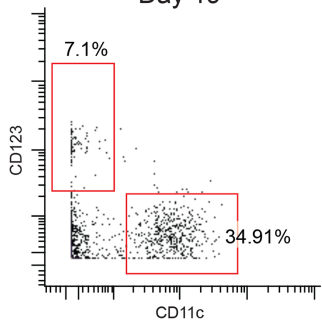

Day 25

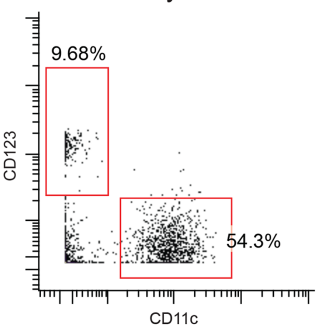

Day 7

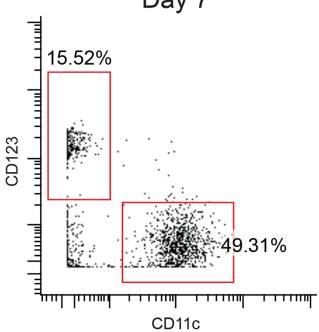

Lin-CD16-CD14-

HLA-DR+CD123+ (pDCs)

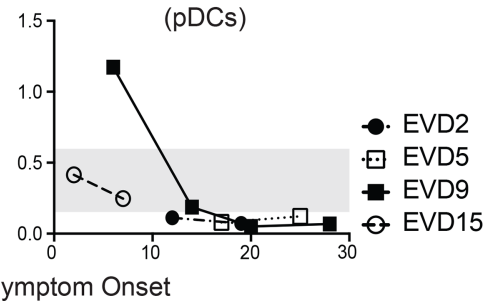

Day 20

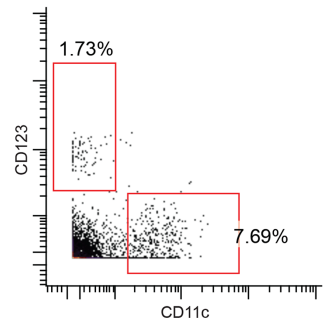

B
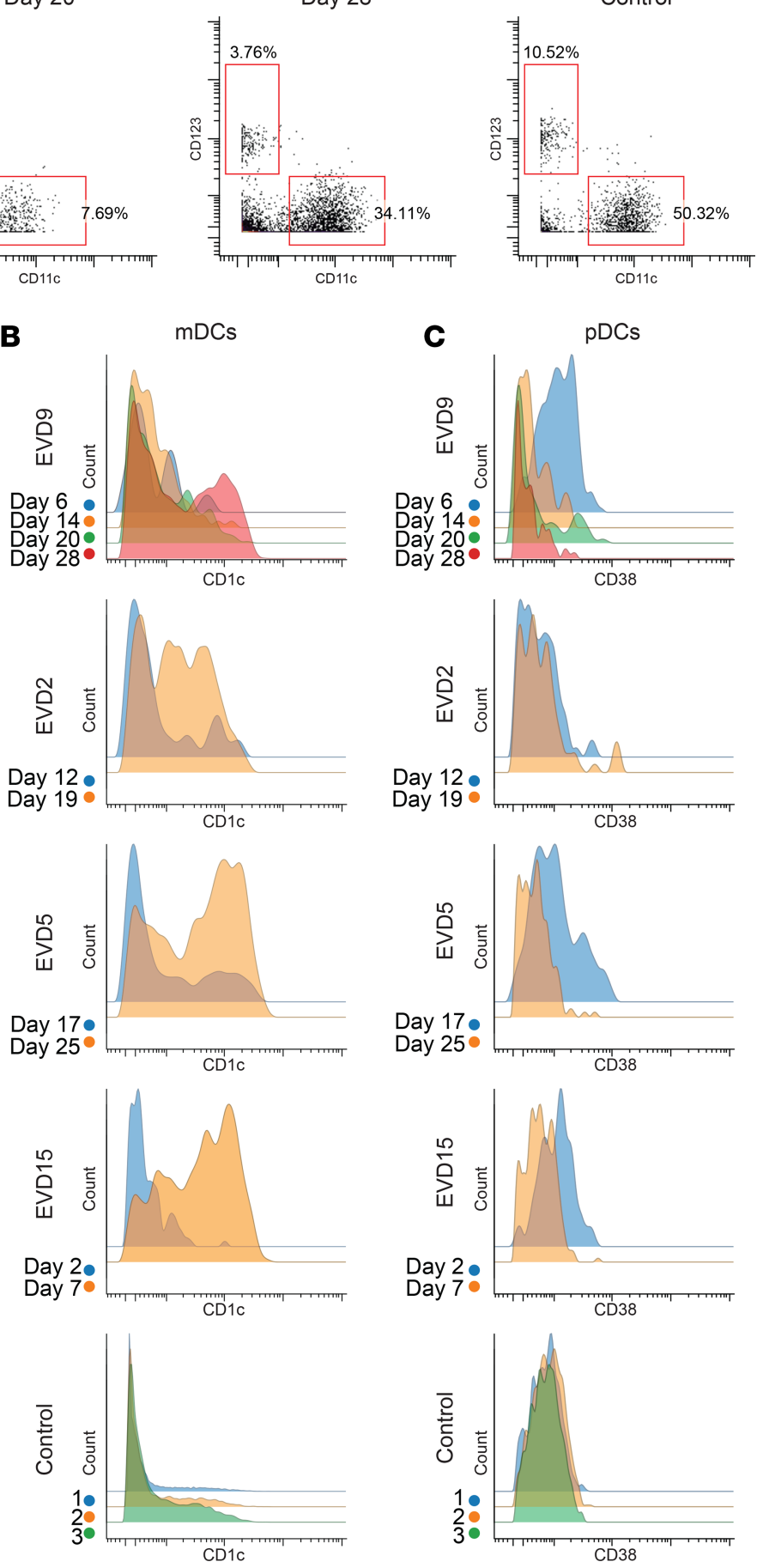

Figure 4. Perturbations in DC populations during EVD. (A) mDCs are lost during the acute phase of disease but return in convalescence. Quantitation of these DCs as a percentage of CD45+CD66- leukocytes is shown in graphs (range for all controls in gray). (B) Coinciding with the return of mDCs in convalescence, CD1c expression on mDCs is dramatically upregulated. (C) pDCs have increased expression of CD38 during the acute-phase time points. Samples from a control obtained on 3 separate dates are shown for comparison for both $\mathbf{B}$ and $\mathbf{C}$.

identified initially in EVD9 and ultimately noted in all 4 patients but, importantly, not in the controls. We hypothesize that these clusters may represent cells of myeloid origin that have egressed from the bone marrow during a time of emergency hematopoiesis. It is well established that physiologic insult, including infection, can lead to expansion of hematopoietic stem cells and myeloid progenitors in order to control an ongoing infection $(50,51)$. This is an important mechanism for myeloid expansion during 
A

Fold change in cell abundance

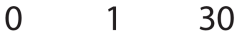

EVD9-Day 14 vs Day 28

EVD2- Day 12 vs Day 19

EVD5- Day 17 vs Day 25

EVD15- Day 2 vs Day7
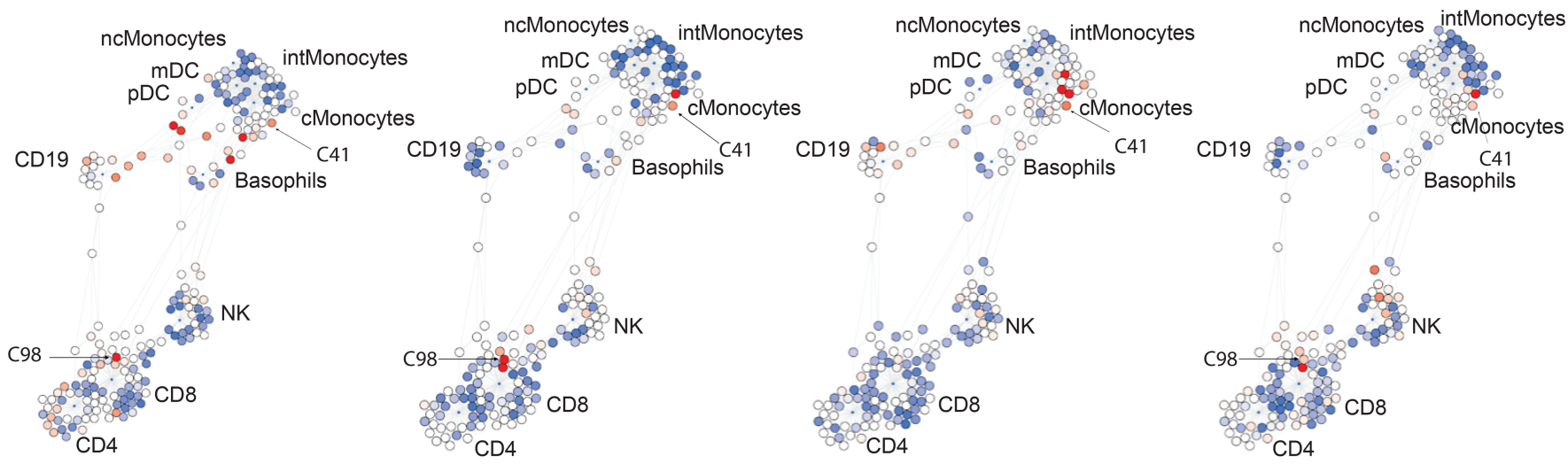

B

Relative difference in median $\mathrm{Ki}-67$ expression

EVD9-Day 14 vs Day 28

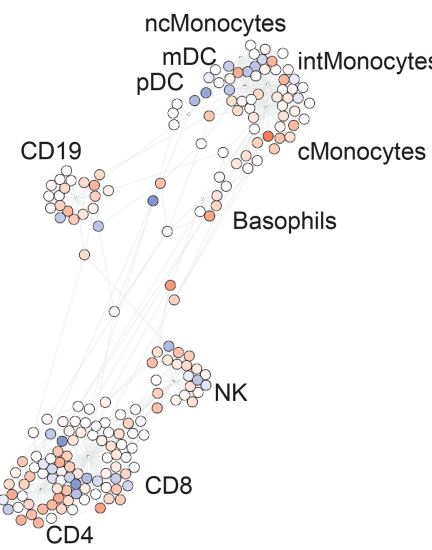

EVD2- Day 12 vs Day 19

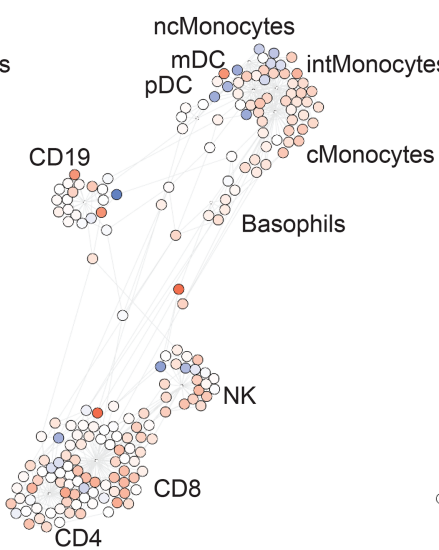

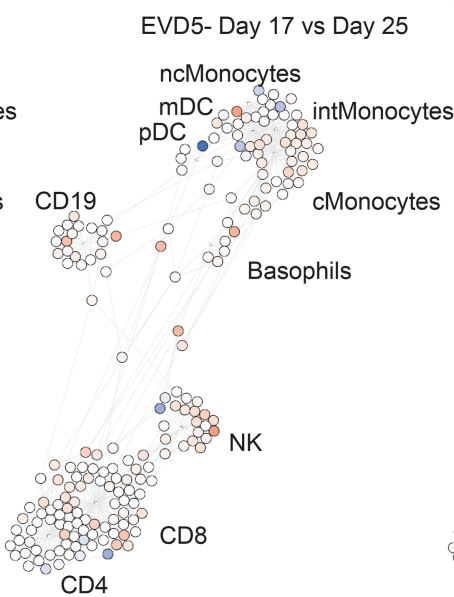

\section{$\begin{array}{lll}-7.5 & 0 & 7.5\end{array}$}

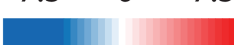

EVD15- Day 2 vs Day7

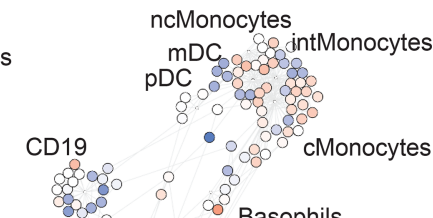

Figure 5. SCAFFoLD maps of cell abundance at acute phase versus convalescent phase. Fold change in cell abundance (A) and differences in median Ki-67 signal (B) at an acute versus a convalescent time point for each patient. Most cell populations, including monocytes, NK cells, and T cells, decrease in abundance during the acute phase but show active proliferation.

inflammation, since, unlike for lymphoid cells, there is no receptor specificity to drive proliferation. Instead, growth factors, such as M-CSF, GM-CSF, and G-CSF, are produced and stimulate the bone marrow to increase myelopoiesis. Indeed, during EVD, all 3 growth factors are produced at higher levels than in normal healthy controls; furthermore, elevated levels of MCSF were associated with severe manifestations of EVD (8). The $\operatorname{Lin}^{-} \mathrm{CD}_{11} \mathrm{~b}^{+}$cells that we observe during acute EVD have similarities to a population of cells known as myeloid-derived suppressor cells (MDSCs) that have been described in sepsis (52) and arise as a result of emergency hematopoiesis. In fact, these MDSCs are reported to produce IL-10 (which is also elevated in EVD, especially in fatal cases) and TGF- $\beta$ to exert immunosuppressive effects. In many cases of fatal EVD, patients die from the disease before generating significant adaptive immune responses, and the immunosuppressive milieu generated by MDSCs could explain these findings. The clinical course of patient EVD9 mirrors this: the patient had very high levels of MCSF from 6 to14 days after symptom onset accompanied by high levels of IL-10 during the same time period as noted in a prior publication (8). This also coincides with the high frequency of the $\mathrm{Lin}^{-} \mathrm{CD} 11 \mathrm{~b}^{+}$cell population, which peaked on day 14 and constituted $17 \%$ of the total non-neutrophil $\mathrm{CD} 45^{+}$events; by day 20 , this population decreased to $1.6 \%$ (it was less than $0.5 \%$ in controls). These findings suggest that, at least in cases of severe EVD, emergency hematopoiesis could be activated. 
pDCs as a solution to the EVD IFN paradox. EBOV encodes potent IFN antagonists that completely block IFN production in vitro (53), though IFN can be detected in the blood of patients (54). Despite these IFN-antagonistic activities of EBOV, some patients manage to mount adaptive immune responses and survive the infection even without therapeutic interventions. This apparent paradox can be explained by the action of pDCs, which are predominantly responsible for generating type I IFN and can present antigens to induce adaptive immunity (22). Important groundwork was laid when Leung et al. demonstrated that pDCs were not susceptible to the IFN-antagonistic activity of VP35 and could be stimulated to generate IFN- $\alpha$ via TLR7 sensing (21). The same authors also demonstrate that $\mathrm{pDCs}$ isolated from human blood were refractory to EBOV infection in vitro (20). This led to the conclusion that pDCs could not be activated by EBOV since they were not infected. However, in this study, we demonstrate that during EBOV infection of humans, pDCs upregulate CD38. CD38 is a well-known activation marker on B and T cells, but less has been reported about its role on pDCs. CD38 is a transmembrane glycoprotein that generates adenine dinucleotides to mobilize intracellular calcium; its expression on DCs is critical for DC mobility and T cell priming (55). CD38 can also mediate interactions with endothelial CD31 (PECAM) to facilitate adhesion and migration (56). Work by Mcllwain et al. showed that CD38 signaling on pDCs is responsible for IFN- $\alpha$ production by $\mathrm{pDCs}$ (unpublished observation). These data suggest that $\mathrm{CD} 38^{+} \mathrm{pDCs}$ are active during EVD in humans. Unlike mDCs, they are not markedly decreased in number during EBOV infection, supporting the hypothesis that $\mathrm{pDCs}$ are active and functional during EVD and able to support the downstream production of adaptive immunity, which is also well established to occur in humans. It will be important to determine whether pDC activity is different in patients who succumb to disease, and these cells could be an important clinical target for immunotherapeutic interventions.

This work clearly has limitations. Clinical samples were not obtained from all patients throughout the course of infection, the overall sample size is very small, and the 4 patients studied received a myriad of experimental therapeutics that could have altered their clinical and immunologic course. As with many studies in humans, only the blood was sampled, so it is impossible to know how tissue levels of these various immune cell populations changed over time. This study also does not address which immune cells are infected in vivo in humans. The comparator population was normal healthy individuals rather than patients with a sepsis-like picture, which might have provided a broader comparison. However, despite these limitations, this work provides what we believe to be novel observations regarding the consequences of EBOV infection on the human immune response in vivo. Most importantly, this complex, multiparameter analysis cannot be exhaustively evaluated in the present article; therefore, the raw data are available to the scientific community (see Methods) to allow for further study and potential insights into the human immune response during EVD.

In conclusion, we present an assessment of the immune response in peripheral blood to EBOV infection in humans using CyTOF. Notable findings include acute loss of monocyte subsets and mDCs, persistence and activation of pDCs, evidence for emergency hematopoiesis, and marked proliferation activity across many immune cell populations.

\section{Methods}

Safety. All work with infectious materials was performed in the BSL-4 containment space of the CDC.

Sample processing. Whole blood collected in EDTA tubes was centrifuged $500 \mathrm{~g}$ for 5 minutes, and plasma was removed. Packed RBCs and leukocytes were suspended in an equal volume of PBS and layered on top of an equal volume of Ficoll 1077 (MilliporeSigma). Following centrifugation at $500 \mathrm{~g}$ for 30 minutes without a brake, the PBMC layer was collected and washed in PBS. Following another centrifugation at $500 \mathrm{~g}$ for 5 minutes, the PBMC pellet was suspended in freezing media ( $90 \% \mathrm{FBS}, 10 \% \mathrm{DMSO}$ ) and aliquoted to cryovials at an equivalent of $2 \mathrm{~mL}$ whole blood per tube.

Processing for mass cytometry. For mass cytometry analysis, cryopreserved PBMC samples were thawed and washed in PBS. For experimental samples, the entire tube of PBMCs (equivalent to $2 \mathrm{~mL}$ whole blood) was used. For control samples, $2 \times 10^{6}$ PBMCs were used. To label dead cells, samples were incubated for 5 minutes with $200 \mu \mathrm{L}$ Cell-ID Cisplatin (Fluidigm) diluted 1:1000 in PBS. Samples were then washed in cell staining medium (CSM) consisting of low-barium PBS with $0.5 \%$ BSA and $0.02 \%$ sodium azide and barcoded using a Cell-ID 20-Plex Pd Barcoding Kit (Fluidigm). Samples were treated with $1 \mathrm{~mL}$ of $1 \times$ Maxpar Fix I Buffer for 10 minutes at room temperature (RT), then washed twice in $1 \mathrm{~mL}$ of $1 \times$ Maxpar Barcode Perm Buffer. Each sample was then incubated with a 
unique barcode for 30 minutes at RT. Samples were washed twice in 1× Maxpar Cell Staining Buffer, suspended in CSM, combined into a single tube, and filtered through a 70- $\mu \mathrm{m}$ filter to remove debris. Human TruStain FcX (BioLegend) was added ( $2.5 \mu \mathrm{L}$ per sample) and incubated for 10 minutes at RT. Surface stains (see Supplemental Table 1 for antibodies used) were then directly added and incubated for 30 minutes at RT with periodic rotation. Samples were washed twice in CSM before the cell pellet was incubated in BD Fix/Perm for 20 minutes at RT. At this point, the samples were removed from BSL-4 containment. Samples were washed twice in CSM; pellets were suspended in $100 \%$ ice-cold methanol and stored overnight at $-80^{\circ} \mathrm{C}$. The following day, samples were washed 3 times in CSM and incubated with intracellular stains (see Supplemental Table 1) for 1 hour at RT. Samples were then washed in CSM and incubated for 20 minutes at RT in an 191/193 Ir DNA Intercalator (DVS Sciences) diluted 1:5000 in PBS with 1.6\% paraformaldehyde. Samples were washed 3 times in MilliQ water, then suspended in $0.1 \times$ EQ beads (Fluidigm) to a concentration of 1000 stained cells $/ \mu \mathrm{L}$. Events were acquired on a CyTOF 2 Mass Cytometer (Fluidigm).

Data processing. FCS files obtained from CyTOF were normalized (57) and de-barcoded (58) using standard protocols, and analyzed in CellEngine (Primity Bio). A representative gating strategy (from a single donor) used to identify cell populations is shown in Figure 1. Frequencies of each cell population were determined as a function of total $\mathrm{CD} 45^{+} \mathrm{CD} 66^{-}$events (live, singlet, non-RBC, and non-platelet). Exported frequencies were plotted in Prism (GraphPad) to generate graphs shown in Figures 2-4. Median signal intensity data for all intracellular signaling markers (phospho-antibodies) were exported to Excel (Microsoft). Fold difference in the experimental sample versus the average median of simultaneously acquired control samples was determined, and the data were plotted in heatmap format using Prism to show each patient's time points and all of the measured cell types (Supplemental Figure 1).

For SCAFFoLD (34) analysis, the $\mathrm{CD} 45^{+} \mathrm{CD} 66^{-}$population was exported as.fcs files and subsampled to 19,000 events per donor and time point (the greatest common denominator among all FCS files). Events were pooled and designated to one of 200 clusters based on their surface marker expression. Landmark nodes were defined by known, gated cell populations from control donors.

Due to the limited sample size, statistical analysis was inappropriate and was not performed.

Study approval. The patients ( 2 female, 2 male) were cared for in the Emory Serious Communicable Diseases Unit. IRB approval was obtained from both Emory University (IRB0076700) and the CDC (IRB6643) for the reported studies. PBMCs were obtained from healthy control donors via an approved IRB protocol (CDC1652). All study participants provided written informed consent and were assigned an EVD number. These same designations were used in prior publications $(8,23)$.

Data availability. The raw.fcs files generated during this study will be made available to investigators upon request.

\section{Author contributions}

AK McElroy designed and conducted experiments, analyzed data, and wrote and edited the manuscript. RSA designed and conducted experiments, analyzed data, and edited the manuscript. DRM designed experiments, analyzed data, and edited the manuscript. HC analyzed data and edited the manuscript. ZBH analyzed data and edited the manuscript. NM conducted experiments. AK Mehta obtained IRB approval, enrolled patients, and edited the manuscript. GN, STN, and CFS supervised the study and edited the manuscript.

\section{Acknowledgments}

We would like to thank the patients for their willingness to participate and acknowledge the medical staff of the Emory University Serious Communicable Diseases Unit for providing excellent care to the patients. We thank Tanya Klimova for editing the manuscript. The findings and conclusions in this report are those of the authors and do not necessarily represent the official position of the US Centers for Disease Control and Prevention or the US Food and Drug Administration. AK McElroy was supported by a Burroughs Wellcome award (no. 1013362.01) and an NIH K08 grant (AI119448) during the course of this work. RSA was supported by Cooperative Centers on Human Immunology (CCHI) Opportunity funding 2016-2017. ZBH, DRM, HC, NM, GN were supported by US Food and Drug Administration contracts HHSF223201610018C and HHSF223201210194C. The Defense Advanced Research Projects Agency (W31P4Q-14-1-0010) partially supported this work. 
Address correspondence to: Anita K. McElroy, Division of Pediatric Infectious Disease and Center for Vaccine Research, University of Pittsburgh School of Medicine, 3501 Fifth Ave, BST3 9050, Pittsburgh, Pennsylvania 15206, USA. Phone: 412.648.4026; Email: mcelroya@pitt.edu.

1. Cimini E, et al. Different features of V $\delta 2 \mathrm{~T}$ and NK cells in fatal and non-fatal human Ebola infections. PLoS Negl Trop Dis. 2017;11(5):e0005645.

2. Lüdtke A, et al. Ebola virus disease is characterized by poor activation and reduced levels of circulating CD16+ monocytes J Infect Dis. 2016;214(suppl 3):S275-S280.

3. Speranza E, et al. T-cell receptor diversity and the control of T-cell homeostasis mark ebola virus disease survival in humans. J Infect Dis. 2018;218(suppl 5):S508-S518.

4. Ruibal P, et al. Unique human immune signature of Ebola virus disease in Guinea. Nature. 2016;533(7601):100-104.

5. Liu X, et al. Transcriptomic signatures differentiate survival from fatal outcomes in humans infected with Ebola virus. Genome Biol. 2017;18(1):4.

6. McElroy AK, et al. Biomarker correlates of survival in pediatric patients with Ebola virus disease. Emerging Infect Dis. 2014;20(10):1683-1690.

7. McElroy AK, et al. Ebola hemorrhagic fever: novel biomarker correlates of clinical outcome. J Infect Dis. 2014;210(4):558-566

8. McElroy AK, et al. Kinetic analysis of biomarkers in a cohort of US patients with Ebola virus disease. Clin Infect Dis. 2016;63(4):460-467.

9. Baize S, et al. Defective humoral responses and extensive intravascular apoptosis are associated with fatal outcome in Ebola virus-infected patients. Nat Med. 1999;5(4):423-426.

10. Wauquier N, Becquart P, Padilla C, Baize S, Leroy EM. Human fatal zaire ebola virus infection is associated with an aberrant innate immunity and with massive lymphocyte apoptosis. PLoS Negl Trop Dis. 2010;4(10):e837.

11. Li J, et al. Age and Ebola viral load correlate with mortality and survival time in 288 Ebola virus disease patients. Int J Infect Dis. 2016;42:34-39.

12. Sanchez A, et al. Analysis of human peripheral blood samples from fatal and nonfatal cases of Ebola (Sudan) hemorrhagic fever: cellular responses, virus load, and nitric oxide levels. J Virol. 2004;78(19):10370-10377.

13. Ksiazek TG, et al. Clinical virology of Ebola hemorrhagic fever (EHF): virus, virus antigen, and IgG and IgM antibody findings among EHF patients in Kikwit, Democratic Republic of the Congo, 1995. J Infect Dis. 1999;179(suppl 1):S177-S187.

14. Geisbert TW, et al. Pathogenesis of Ebola hemorrhagic fever in cynomolgus macaques: evidence that dendritic cells are early and sustained targets of infection. Am J Pathol. 2003;163(6):2347-2370.

15. Ströher U, West E, Bugany H, Klenk HD, Schnittler HJ, Feldmann H. Infection and activation of monocytes by Marburg and Ebola viruses. J Virol. 2001;75(22):11025-11033.

16. Gupta M, Mahanty S, Ahmed R, Rollin PE. Monocyte-derived human macrophages and peripheral blood mononuclear cells infected with Ebola virus secrete MIP-1alpha and TNF-alpha and inhibit poly-IC-induced IFN-alpha in vitro. Virology. 2001;284(1):20-25.

17. Olejnik J, et al. Ebolaviruses associated with differential pathogenicity induce distinct host responses in human macrophages. J Virol. 2017;91(11):e00179-17.

18. Bosio CM, et al. Ebola and Marburg viruses replicate in monocyte-derived dendritic cells without inducing the production of cytokines and full maturation. J Infect Dis. 2003;188(11):1630-1638.

19. Mahanty S, Hutchinson K, Agarwal S, McRae M, Rollin PE, Pulendran B. Cutting edge: impairment of dendritic cells and adaptive immunity by Ebola and Lassa viruses. J Immunol. 2003;170(6):2797-2801.

20. Leung LW, Martinez O, Reynard O, Volchkov VE, Basler CF. Ebola virus failure to stimulate plasmacytoid dendritic cell interferon responses correlates with impaired cellular entry. J Infect Dis. 2011;204(suppl 3):S973-S977.

21. Leung LW, Park MS, Martinez O, Valmas C, López CB, Basler CF. Ebolavirus VP35 suppresses IFN production from conventional but not plasmacytoid dendritic cells. Immunol Cell Biol. 2011;89(7):792-802.

22. Swiecki M, Colonna M. The multifaceted biology of plasmacytoid dendritic cells. Nat Rev Immunol. 2015;15(8):471-485.

23. McElroy AK, et al. Human Ebola virus infection results in substantial immune activation. Proc Natl Acad Sci U S A. 2015;112(15):4719-4724.

24. Bradfute SB, et al. Lymphocyte death in a mouse model of Ebola virus infection. J Infect Dis. 2007;196(suppl 2):S296-S304.

25. Bradfute SB, et al. Mechanisms and consequences of Ebolavirus-induced lymphocyte apoptosis. J Immunol. 2010;184(1):327-335.

26. Reed DS, Hensley LE, Geisbert JB, Jahrling PB, Geisbert TW. Depletion of peripheral blood T lymphocytes and NK cells during the course of ebola hemorrhagic fever in cynomolgus macaques. Viral Immunol. 2004;17(3):390-400.

27. Gupta M, Spiropoulou C, Rollin PE. Ebola virus infection of human PBMCs causes massive death of macrophages, CD4 and CD8 T cell sub-populations in vitro. Virology. 2007;364(1):45-54.

28. Iampietro M, et al. Ebola virus glycoprotein directly triggers T lymphocyte death despite of the lack of infection. PLoS Pathog. 2017;13(5):e1006397.

29. Wauquier N, Padilla C, Becquart P, Leroy E, Vieillard V. Association of KIR2DS1 and KIR2DS3 with fatal outcome in Ebola virus infection. Immunogenetics. 2010;62(11-12):767-771.

30. Davis CW, et al. Longitudinal analysis of the human B cell response to Ebola virus infection. Cell. 2019;177(6):1566-1582.e17.

31. Ellebedy AH, et al. Defining antigen-specific plasmablast and memory B cell subsets in human blood after viral infection or vaccination. Nat Immunol. 2016;17(10):1226-1234.

32. Bandura DR, et al. Mass cytometry: technique for real time single cell multitarget immunoassay based on inductively coupled plasma time-of-flight mass spectrometry. Anal Chem. 2009;81(16):6813-6822.

33. Alcántara-Hernández M, et al. High-dimensional phenotypic mapping of human dendritic cells reveals interindividual variation and tissue specialization. Immunity. 2017;47(6):1037-1050.e6. 
34. Bendall SC, et al. Single-cell mass cytometry of differential immune and drug responses across a human hematopoietic continuum. Science. 2011;332(6030):687-696.

35. Elhmouzi-Younes J, et al. In depth comparative phenotyping of blood innate myeloid leukocytes from healthy humans and macaques using mass cytometry. Cytometry A. 2017;91(10):969-982.

36. Ferreira VH, Kumar D, Humar A. Deep profiling of the CD8+ T-cell compartment identifies activated cell subsets and multifunctional responses associated with control of cytomegalovirus viremia. Transplantation. 2019;103(3):613-621.

37. Bengsch B, et al. Epigenomic-guided mass cytometry profiling reveals disease-specific features of exhausted CD8 T cells. Immunity. 2018;48(5):1029-1045.e5

38. Doyle $\mathrm{EH}$, et al. Individual liver plasmacytoid dendritic cells are capable of producing IFN $\alpha$ and multiple additional cytokines during chronic HCV infection. PLoS Pathog. 2019;15(7):e1007935.

39. Liddell AM, et al. Characteristics and clinical management of a cluster of 3 patients with Ebola virus disease, including the first domestically acquired cases in the United States. Ann Intern Med. 2015;163(2):81-90.

40. Lyon GM, et al. Clinical care of two patients with Ebola virus disease in the United States. NEngl J Med. 2014;371(25):2402-2409.

41. Uyeki TM, et al. Clinical management of Ebola virus disease in the United States and Europe. N Engl J Med. 2016;374(7):636-646.

42. Kraft CS, et al. The use of TKM-100802 and convalescent plasma in 2 patients with Ebola virus disease in the United States. Clin Infect Dis. 2015;61(4):496-502.

43. Bjornson-Hooper ZB, Fragiadakis GK, Spitzer MH, Madhireddy D, McIlwain D, Nolan GP. A comprehensive atlas of immunological differences between humans, mice and non-human primates [preprint]. https://doi.org/10.1101/574160. Posted on bioRxiv March 11, 2019.

44. Bjornson-Hooper ZB, et al. Cell type-specific monoclonal antibody cross-reactivity screening in non-human primates and development of comparative immunophenotyping panels for CyTOF [preprint]. https://doi.org/10.1101/577759. Posted on bioRxiv March 14, 2019

45. Spitzer $\mathrm{MH}$, et al. An interactive reference framework for modeling a dynamic immune system. Science. 2015;349(6244):1259425.

46. Overgaard NH, Jung JW, Steptoe RJ, Wells JW. CD4+/CD8+ double-positive T cells: more than just a developmental stage? J Leukoc Biol. 2015;97(1):31-38.

47. Sampath P, Moideen K, Ranganathan UD, Bethunaickan R. Monocyte subsets: phenotypes and function in tuberculosis infection. Front Immunol. 2018;9:1726.

48. Ong SM, et al. A novel, five-marker alternative to CD16-CD14 gating to identify the three human monocyte subsets. Front Immunol. 2019;10:1761.

49. Michlmayr D, et al. Comprehensive innate immune profiling of chikungunya virus infection in pediatric cases. Mol Syst Biol. 2018;14(8):e7862.

50. Furusawa J, et al. Promotion of expansion and differentiation of hematopoietic stem cells by interleukin-27 into myeloid progenitors to control infection in emergency myelopoiesis. PLoS Pathog. 2016;12(3):e1005507.

51. Loftus TJ, Mohr AM, Moldawer LL. Dysregulated myelopoiesis and hematopoietic function following acute physiologic insult. Curr Opin Hematol. 2018;25(1):37-43

52. Schrijver IT, Théroude C, Roger T. Myeloid-derived suppressor cells in sepsis. Front Immunol. 2019;10:327.

53. Messaoudi I, Amarasinghe GK, Basler CF. Filovirus pathogenesis and immune evasion: insights from Ebola virus and Marburg virus. Nat Rev Microbiol. 2015;13(11):663-676.

54. Villinger F, et al. Markedly elevated levels of interferon (IFN)-gamma, IFN-alpha, interleukin (IL)-2, IL-10, and tumor necrosis factor-alpha associated with fatal Ebola virus infection. J Infect Dis. 1999;179(suppl 1):S188-S191.

55. Partidá-Sánchez S, Rivero-Nava L, Shi G, Lund FE. CD38: an ecto-enzyme at the crossroads of innate and adaptive immune responses. Adv Exp Med Biol. 2007;590:171-183.

56. Quarona V, et al. CD38 and CD157: a long journey from activation markers to multifunctional molecules. Cytometry B Clin Cytom. 2013;84(4):207-217.

57. Finck R, et al. Normalization of mass cytometry data with bead standards. Cytometry A. 2013;83(5):483-494.

58. Zunder ER, et al. Palladium-based mass tag cell barcoding with a doublet-filtering scheme and single-cell deconvolution algorithm. Nat Protoc. 2015;10(2):316-333. 